reduction persisted for up to $20+$ years after the ovarian tumor diagnosis.

Conclusion The risk of endometrial cancer was decreased beyond the first year after a benign ovarian tumor and the decrease persisted for 20 or more years. The possible underlying mechanisms are not known and should be investigated further.

Disclosures None.

\section{THE ONCOLOGIC OUTCOME AFTER FERTILITY-SPARING HORMONAL MANAGEMENT MORE THAN 9 MONTHS TREATMENT FOR EARLY STAGE ENDOMETRIOID ENDOMETRIAL CANCER}

Su Hyun Chae, Seung-Hyuk Shim, Sun Joo Lee. Konkuk Medical Center

\subsection{6/ijgc-2020-ESG0.52}

Introduction/Background Hormonal management is an alternative treatment for preserving fertility in patients with early stage endometrioid endometrial cancer (EC). The safety and clinical outcome in longer treatment more than 9 months has controversial. This study aimed to define the oncologic outcomes after hormone therapy more than 9 months for endometrioid EC.

Methodology We retrospectively analyzed patients presumed to have stage IA, grade 1-2 endometrioid EC who underwent fertility-sparing treatment. Concurrent medroxyprogesterone (MPA) and levonorgestrel-release intrauterine devices were used for treatment. The remission rate and progression free survival were analysed each of the short term treatment who had treatment under 9 months and long term groups who had treatment duration over 9 months.

Results One hundred twenty patients presumed to have stage IA, grade 1 endometrioid EC had treated with hormonal medication for fertility sparing. The median age was 33.5 (range 22-43) years old and the median treatment duration was 10.7 (3-102) months. The Complete remission (CR) rate was $84.2 \%(101 / 120)$ and the median time interval to CR was 9.3 (2-84) months. The median follow-up time was 32.9 (1-130) months. The recurrence rate was $31.7 \%$ (38/120) and the median time to recurrence was 11 (1-92) months. The cumulative CR rate by $3,6,9,12,15,18,24$ months was $21.7 \%$, $36.7 \%, 50.8 \%, 61.7 \%, 70.8 \%, 74.2 \%$, and $78.3 \%$ respectively. The CR rates in group A and B were $86.7 \%$ and $82.7 \%$ in group $\mathrm{A}$ and $\mathrm{B}$. The recurrence rates in two group wrere $35.6 \%$ and $29.3 \%$, respectively.

Conclusion Fertility sparing treatment with high dose progestin over 9 months in early stage endometrioid EC has showed high rate of CR. However, medical treatment over 9 months should counsel with patients in detail and oncologists should make careful decision.

Disclosures I have no conflicts of interest to disclose.

\section{THE ADDED VALUE OF SENTINEL NODE MAPPING IN ENDOMETRIAL CANCER}

\footnotetext{
${ }^{1}$ Liron Kogan, ${ }^{1}$ Emad Matanes, ${ }^{2}$ Michel Wissing, ${ }^{1}$ Cristina Mitric, ${ }^{1}$ Shannon Salvador, ${ }^{1}$ Susie Lau, ${ }^{3}$ Walter Gotlieb. 'Mcgill University; Division of Gynecologic Oncology, Jewish General Hospital; ${ }^{2}$ Mcgill University; Division of Cancer Epidemiology, Department of Oncology; ${ }^{3}$ Jewish General Hospital; Mcgill University
}

10.1136/ijgc-2020-ESGO.53
Introduction/Background Endometrial cancer (EC) is the most common gynecological malignancy worldwide, with an estimated 382,069 new cases and 89,929 deaths in 2018. Lymph node involvement represents one of the most important prognostic factors and guides better planning of post-operative adjuvant treatment. Whereas lymph node assessment has been included in surgical staging since 1988, the optimal procedure for lymph node evaluation is controversial, ranging from full pelvic and para-aortic lymph node dissection (LND) to complete omission of LND. We previously evaluated the oncologic outcomes of 472 cases of EC (SLN with LND vs. LND alone) and demonstrated significantly lower likelihood of pelvic sidewall recurrences in patients who underwent SLN. These data raised the possibility that addition of SLN biopsy may not just be equivalent to conventional staging but may actually increase the detection of metastatic disease, resulting in better stratification of patients into risk groups, optimal adjuvant therapy prescription and as a result, better oncologic outcomes. In this study, we investigated the long-term oncological outcome of adding SLN to pelvic LND in patients with EC.

Methodology Retrospective study comparing survival outcomes (overall survival (OS), disease-specific survival (DSS), progression-free survival (PFS), recurrence-free survival) between endometrial cancer patients undergoing surgical staging, which included LND with or without SLN in non-overlapping contiguous eras. Hazard ratios (HR) and their respective 95\% confidence intervals $(95 \% \mathrm{CI})$ were calculated using Cox proportional hazard models.

Results 193 patients underwent LND and 250 patients had SLN mapping prior to LND. Clinical characteristics, including adjuvant therapy use, were similar between groups. During a median follow-up period of 6.9 years, addition of SLN was associated with more favorable oncological outcomes compared to LND with 6-year OS of $90 \%$ compared to $81 \%$ $(p=0.009)$, and PFS of $85 \%$ compared to $75 \% \quad(p=0.01)$ respectively. SLN was associated with improved OS (HR 0.5, 95\% CI 0.3-0.8, p=0.004), DSS (HR 0.5, 95\%CI 0.2-1.0, $\mathrm{p}=0.05$ ) and PFS (HR 0.6, 95\% CI $0.4-0.9, \mathrm{p}=0.03$ ) in a multivariable analysis as well, adjusted for age, ASA score, stage, grade, non-endometrioid histology, and LVSI. Patients who were staged with SLN were less likely to have a recurrence in the pelvis or lymph node basins compared to patients who underwent LND only (6-year recurrence-free survival 95\% vs 90\%, p=0.04).

Conclusion Addition of SLN was associated with improved clinical outcomes compared to LND alone in patients with endometrial cancer undergoing surgical staging.

Disclosures We have no disclosures.

\section{OMITTING LYMPHADENECTOMY IN OBESE ENDOMETRIAL CANCER PATIENTS UNDERGOING SENTINEL LYMPH NODE MAPPING: WHEN MORE IS LESS}

${ }^{1}$ Liron Kogan, ${ }^{1}$ Emad Matanes, ${ }^{1}$ Cristina Mitric, ${ }^{1}$ Shannon Salvador, ${ }^{1}$ Susie Lau, 'Walter Gotlieb. 'Mcgill University; Division of Gynecologic Oncology, Jewish General Hospital; ${ }^{2}$ Jewish General Hospital; Mcgill University

\subsection{6/ijgc-2020-ESG0.54}

Introduction/Background The prevalence of obesity in the United States has tripled over the last 40 years. Obesity is a significant risk factor for endometrial cancer (EC). Sentinel lymph node (SLN) sampling has been applied for EC surgery 